\title{
AN ADDITIVE DECOMPOSITION THEOREM FOR ANALYTIC FUNCTIONS
}

\author{
DONALD E. MYERS
}

Abstract. Kraut, Busenberg, and Hall have shown that the uniqueness of the additive decomposition for functions of several variables given by Bochner is due to the asymptotic properties of the summands. In this paper, the uniqueness theorem is used to extend the class of functions possessing unique decompositions.

Recently Kraut, Busenberg and Hall [2] have observed that the asserted uniqueness for additive decompositions of holomorphic functions on tubes in complex $n$-space as given by Bochner [1] is due to the asymptotic decay of the summands. In this note we will show that [1], [2] actually provide somewhat more general results than are stated. Following [1], a tube $T$ in $C^{n}$ is of the form $\{z \mid z$ $\left.=\left(z_{1}, \cdots, z_{n}\right), \sigma_{i}<R\left(z_{i}\right)<J_{i}\right\}$. A tube is said to be octant shaped if for each $i$ either $\sigma_{i}=-\infty$ or $\mathfrak{J}_{i}=+\infty$ but not both. As observed in [2], Bochner's hypothesis for a unique decomposition is sufficient and the following theorem shows that it is not necessary.

THEOREM. Let $f$ be analytic in a tube $T$ and suppose there exists a point $\alpha=\left(\alpha_{1}, \cdots, \alpha_{n}\right)$ not in the closure of the base of $T$ and an $n$-tuple $\beta=\left(\beta_{1}, \cdots, \beta_{n}\right), 0<\beta_{i}<1$, such that

$$
|f(z)| \prod_{i=1}^{n}\left|z_{i}-\alpha_{i}\right|^{\beta_{i}}<M
$$

for some positive constant $M$. Then there exist unique functions $f_{1}, \cdots$, $f_{2^{n}}$ each analytic in an octant shaped tube such that $f=f_{1}+\cdots+f_{2^{n}}$ in $T$.

Proof. Clearly $g(z)=f(z) / \prod_{i=1}^{n}\left(z_{i}-\alpha_{i}\right)$ satisfies Bochner's condition of being square integrable with respect to the imaginary part for each $z_{i}$. Then $g(z)$ has a decomposition $g=g_{1}+\cdots+g_{2^{n}}$ and hence $f=f_{1}+\cdots+f_{2^{n}}$ where $f_{j}(z)=g_{j}(z) \prod_{i=1}^{n}\left(z_{i}-\alpha_{i}\right)$. It is only necessary then to show that each $f_{j}(z)$ is unique, i.e. independent of $\alpha=\left(\alpha_{1}, \cdots, \alpha_{n}\right)$. By Theorem 1 [2], it is sufficient to show each $f_{j}$ is

Presented to the Society, November 21, 1970 under the title $A n$ additive decomposition theorem; received by the editors June 24, 1970.

AMS 1970 subject classifications. Primary 32A07, 32A25; Secondary 30A84, 30A86.

Key words and phrases. Additive decomposition, tube, domain uniqueness. 
bounded in the octant-shaped tube $T_{j}$ and $f_{j} \rightarrow 0$ as any one of the $x_{j} \rightarrow \pm \infty$ in $T_{j}$.

Since the construction of the summands, as given by Bochner, is typified by the case $n=1$ we will verify the result for $n=1$ and indicate the general case. Let the base of $T$ be $\{x \mid \sigma<x<J\}$. Then $T_{1}$ $=\{z \mid \operatorname{Re} z>\sigma\}, T_{2}=\{z \mid \operatorname{Re} z<J\}$.

$$
\begin{aligned}
f(z) & =(z-\alpha) g(z)=\frac{(z-\alpha)}{2 \Pi i} \int_{C} \frac{[f(w) /(w-\alpha)]}{w-z} d w \\
& =\frac{-1}{2 \Pi i} \int_{C} \frac{f(w)}{w-\alpha} d w+\frac{1}{2 \Pi i} \int_{C} \frac{f(w)}{w-z} d w \\
& =\frac{1}{2 \Pi} \int_{-\infty}^{\infty} \frac{f(J+i v)}{\Im+i v-z} d v+\frac{1}{2 \Pi} \int_{\infty}^{-\infty} \frac{f(\sigma+i v)}{\sigma+i v-z} i d v \\
& =f_{2}(z)+f_{1}(z),
\end{aligned}
$$

since

$$
\frac{1}{2 \Pi i} \int_{J}^{\sigma} \frac{f(u+i t)}{u+i t-z} d u \rightarrow 0 \text { and } \frac{1}{2 \Pi i} \int_{0}^{J} \frac{f(u-i t)}{u-i t-z} d u \rightarrow 0
$$

as $t \rightarrow \infty$ and $\sigma<\operatorname{Re} z<$ J.

$$
\begin{aligned}
\left|f_{2}(z)\right| & =\frac{1}{2 \Pi}\left|\int_{-\infty}^{\infty} \frac{f(\Im+i v)}{\Im+i v-z} d v\right| \\
& \leqq \frac{1}{2 \Pi} \int_{-\infty}^{\infty} \frac{M}{|\Im+i v-z||\Im+i v-\alpha|^{\beta}} d v \\
& \leqq \frac{1}{2 \Pi} \int_{-\infty}^{\infty} \frac{M}{|v|^{1+\beta}} d v<\infty
\end{aligned}
$$

for $\operatorname{Re} z<\mathfrak{J}$, so that $f_{2}$ is bounded and similarly for $f_{1}$. Furthermore, as $x \rightarrow \pm \infty, f_{1}$ and $f_{2}$ respectively tend to zero. The general case merely requires iterations.

\section{REFERENCES}

1. S. Bochner, Bounded analytic functions in several variables and multiple Laplace integrals, Amer. J. Math. 59 (1937), 732.

2. E. Kraut, S. Busenberg and W. Hall, On an additive decomposition of functions several complex variables, Bull. Amer. Math. Soc. 74 (1968), 372-374. MR 36 \#2826.

University of Arizona, Tucson, Arizona 85721 